\title{
On the continuous plug potential profile in a tandem mirror
}

\author{
I. Katanuma, ${ }^{\text {a) }}$ Y. Kiwamoto, Y. Tatematsu, K. Ishii, T. Saito, T. Tamano, \\ and K. Yatsu \\ Plasma Research Center, University of Tsukuba, Ibaraki 305-8577, Japan
}

(Received 23 October 1997; accepted 19 February 1998)

\begin{abstract}
The plug potential is formed experimentally, even without high energy sloshing ion population in the end-mirror cells of a tandem mirror. It has been shown analytically that the plug potential can be formed in the present experimental conditions by using the plausible distribution functions of ions and electrons and a small ionization source. In this paper, the required ionization rate is estimated for the continuous potential profile around the plug by using Emmert's source. (C) 1998 American Institute of Physics. [S1070-664X(98)03805-1]
\end{abstract}

The original scenario of the plug potential formation requires high energy sloshing ions, as well as populations of electrostatically trapped warm electrons and magnetically trapped high energy electrons. ${ }^{1}$ The present tandem mirror experiments, however, form a plug potential without the population of sloshing ions. ${ }^{2,3}$

We have proposed the mechanism of the plug potential formation in the end cell of a tandem mirror. ${ }^{4-6}$ That is, the plug potential is formed by the charge neutrality condition of ions and electrons. Here, some of the ions come from the central cell and others are trapped magnetically in the thermal barrier region and a little amount of ionized ions is included. On the other hand some of the electrons come from the central cell and the remains are trapped electrostatically in the plug region.

At the point of the potential maximum in Fig. 1(a), there is a flow of ions from the central cell. On the other hand the electrons are prevented by the potential from escaping to the end-wall. The situation around the plug region, therefore, is the same as that of the sheath potential formation, which leads to discontinuity of the potential profile.

The formation of the sheath potential in front of the wall has been studied extensively. ${ }^{7}$ Although the sheath formation can be avoided by a plasma flow velocity much like the Bohm sheath condition, ${ }^{8}$ the ion flow velocity is zero at the plug because the plug is the position at the potential maximum. To the authors' knowledge we do not know that the sheath potential exists stably in the region where the plasma is confined experimentally. We believe that the charge neutrality condition holds around the plug, so that we seek the condition that the jump of the potential does not appear.

We have introduced the distribution function of ions, which come from the direction of the end-wall toward the central cell. This population of ions makes the distribution function continuous across the line $\varepsilon=\mu B_{p}+e \phi_{p}$ in the velocity space, which is localized in the gray region pointed out by the arrow with the "continuous distribution function" in Fig. 1(b). Here $\varepsilon, \mu$ are energy and magnetic moment, and $B_{p}, \phi_{p}$ are the heights of the magnetic field and the potential at the plug. This distribution function makes the potential continuous around the plug region. ${ }^{6}$

We have concluded that a small amount of ions which come from the direction of the end-wall plays an important role in the formation of the plug potential without discontinuity. If this "continuous distribution function" in Fig. 1(b) is supplied by the ionization process, the "continuous distribution function" is not realistic because these ions have a high perpendicular energy, which is almost the same as that of passing ions, around the plug. In this paper we assume that the population of ions which come from the direction of the end-wall are supplied by the ionization of neutral atoms. Instead of the "continuous distribution function" in Fig. 1(b) we adopt the Emmert's source ${ }^{9}$ as an ionization source, which is considered to be a plausible source term in the sheath potential formation,

$$
\begin{aligned}
S_{i}(z, \varepsilon, \mu)= & \langle\sigma v\rangle n_{e} n_{0}\left(m_{i}^{2} / 4 \pi T_{n i}^{2}\right) v_{\|}(z, \varepsilon, \mu) \\
& \times \exp \left\{-[\varepsilon-e \phi(z)] / T_{n i}\right\} .
\end{aligned}
$$

Here $\langle\sigma v\rangle$ is the ionization rate coefficient, $n_{e}, n_{0}$ are the densities of the electrons and neutral atoms, respectively, $T_{n i}, m_{i}$ are the temperature and the mass of ionized ions. The variable $v_{\|}$is the velocity along magnetic field line which is given in term of $\varepsilon$ and $\mu$ to be $v_{\|}(z, \varepsilon, \mu)$ $=\sqrt{2\{\varepsilon-\mu B(z)-e \phi(z)\} / m_{i}}$.

The distribution function of ionized ions $f_{\text {ioni }}$ is obtained by the source term (1),

$$
\begin{aligned}
f_{\text {ioni }}(\varepsilon, \mu)= & \int_{z_{p}}^{z} \mathrm{~d} z_{0} \frac{S_{i}\left(z_{0}, \varepsilon, \mu<\mu_{x}\right)}{v_{\|}\left(z_{0}, \varepsilon, \mu\right)} \\
& +\int_{z_{\text {turn }}(\varepsilon, \mu)}^{z} \mathrm{~d} z_{0} \frac{S_{i}\left(z_{0}, \varepsilon, \mu \geqslant \mu_{x}\right)}{v_{\|}\left(z_{0}, \varepsilon, \mu\right)} \\
& +\int_{z}^{z_{m}} \mathrm{~d} z_{0} \frac{S_{i}\left(z_{0}, \varepsilon, \mu \geqslant \mu_{x}\right)}{v_{\|}\left(z_{0}, \varepsilon, \mu\right)} \\
& +\int_{z}^{z_{\text {turn }}(\varepsilon, \mu)} \mathrm{d} z_{0} \frac{S_{i}\left(z_{0}, \varepsilon, \mu<\mu_{x}\right)}{v_{\|}\left(z_{0}, \varepsilon, \mu\right)},
\end{aligned}
$$

where $z_{\text {turn }}$ and $\mu_{x}$ satisfy, respectively,

$$
\varepsilon=\mu B\left(z_{\text {turn }}\right)+e \phi\left(z_{\text {turn }}\right), \quad \mu_{x}=\frac{e \phi(z)-e \phi\left(z_{0}\right)}{B\left(z_{0}\right)-B(z)} .
$$

Here $z_{p}, z_{m}$ are the axial coordinates of the plug and outer mirror throat, respectively, and $z_{0}$ is the axial position where 
(a)

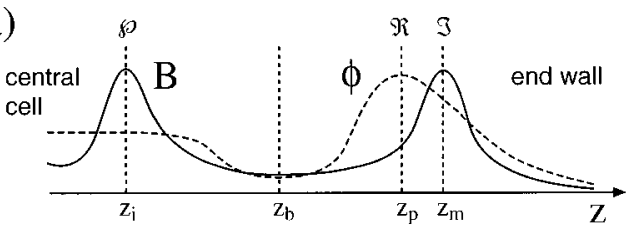

(b)

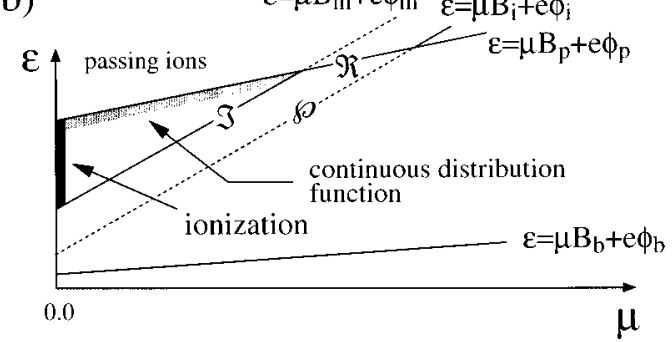

FIG. 1. Schematic diagram of the end-mirror cell of a tandem mirror. (a) is the axial magnetic and electrostatic potential profiles. (b) is the ion velocity space described by $\varepsilon$ and $\mu$. Here the symbols $\wp, \mathfrak{R}, \mathfrak{I}$ are the lines $\varepsilon$ $=\mu B_{i}+e \phi_{i}, \varepsilon=\mu B_{p}+e \phi_{p}, \varepsilon=\mu B_{m}+e \phi_{m}$, respectively, in (b). These lines symbolized by $\wp, \mathfrak{R}, \mathfrak{I}$ in (b) correspond to the ion velocities the parallel component of which, $v_{\|}$, is zero at $z=z_{i}, z=z_{p}, z=z_{m}$, respectively in (a).

ions are born. The distribution function $f_{\text {ioni }}$ is localized in the dark painted region which is pointed by an arrow with "ionization," in the velocity space in Fig. 1(b). So, the ion distribution is discontinuous across the line $\varepsilon=\mu B_{p}+e \phi_{p}$.

The ion distribution function in the other region of velocity space is given, respectively,

$$
f_{i}=n_{i c}\left(m_{i} / 2 \pi T_{i}\right)^{3 / 2} \exp \left\{-\left(\varepsilon-e \phi_{i}\right) / T_{i}\right\},
$$

in the region $v_{\|} \geqslant 0$ and $\varepsilon \geqslant \mu B_{i}+e \phi_{i}$ and $\varepsilon \geqslant \mu B_{p}+e \phi_{p}$, and

$$
f_{i}=n_{i c}\left(m_{i} / 2 \pi T_{i}\right)^{3 / 2} \exp \left\{\left(\varepsilon-e \phi_{i}-\alpha_{i} \mu B_{i}\right) /\left(\alpha_{i}-1\right) T_{i}\right\},
$$

in the region $\varepsilon<\mu B_{i}+e \phi_{i}$ and $\varepsilon \geqslant \mu B_{p}+e \phi_{p}$. The distribution function of the Yushmanov trapped ion between $z_{p}$ $\leqslant z \leqslant z_{m}$ is

$$
\begin{aligned}
f_{i}= & n_{i p}\left(m_{i} / 2 \pi T_{i}\right)^{3 / 2} \exp \left\{\left(\varepsilon-e \phi_{p}-\alpha_{x} \mu B_{p}\right) /\left(\alpha_{x}\right.\right. \\
& \left.-1) T_{i}\right\},
\end{aligned}
$$

where $n_{i p} \equiv n_{i c} \exp \left\{-e\left(\phi_{p}-\phi_{i}\right) / T_{i}\right\}$. Here suffixes $i, m, p$ represent the quantity at the inner mirror throat, outer mirror throat and plug, respectively, as shown in Fig. 1(a).

The electron distribution function $f_{e}$ is assumed to be

$$
f_{e}=n_{e b}\left(m_{e} / 2 \pi T_{e}\right)^{3 / 2} \exp \left\{-\left(\varepsilon+e \phi_{b}\right) / T_{e}\right\},
$$

in the region $\varepsilon \geqslant \mu B_{b}-e \phi_{b}$, and

$$
\begin{aligned}
f_{e}= & n_{e b}\left(m_{e} / 2 \pi T_{e}\right)^{3 / 2} \\
& \times \exp \left\{-\left(\varepsilon+e \phi_{b}-\alpha_{e} \mu B_{b}\right) /\left(1-\alpha_{e}\right) T_{e}\right\},
\end{aligned}
$$

in the region $\varepsilon<\mu B_{b}-e \phi_{b}$, where $n_{e b} \equiv n_{i c} \exp \left\{e\left(\phi_{b}\right.\right.$ $\left.\left.-\phi_{i}\right) / T_{e}\right\}$, and the suffix $b$ represents the quantity at the thermal barrier region. Here the electron distribution functions (7) and (8) are applied in the region $z_{b} \leqslant z \leqslant z_{m}$. The electrons which come from the central cell are assumed to be Maxwellian. The fundamental electron cyclotron resonance
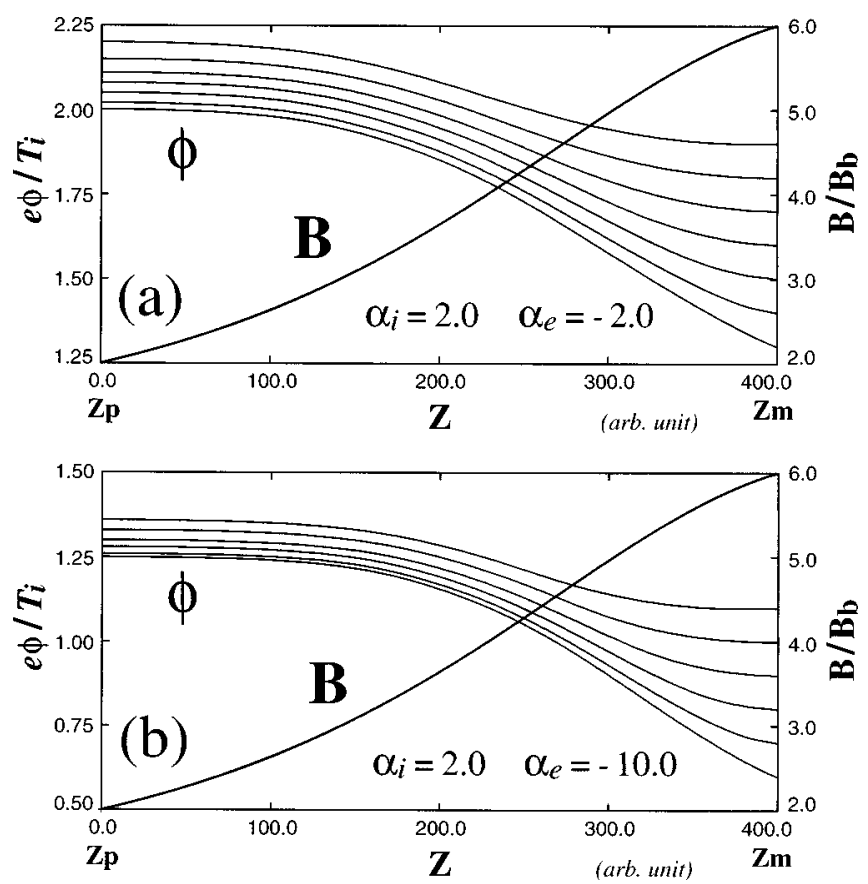

FIG. 2. The axial potential profiles in the mirror cell. Here $B_{b}$ is the magnetic field at the thermal barrier region. The potential profiles from plug $\left(B / B_{b}=2\right)$ to the outer mirror throat are calculated. (a) is the case $\alpha_{i}=2.0$, $\alpha_{e}=-2.0$. (b) is the case $\alpha_{i}=2.0, \alpha_{e}=-10.0$.

heating ( $\left.\omega_{c e} \mathrm{ECRH}\right)$ is applied at the point $B / B_{b}=2$, so that the electrons trapped in the plug potential have a nonMaxwellian distribution in (8).

Figure 2 shows the axial potential profiles calculated on the assumption of the charge neutrality condition by using the ion and electron distribution functions. The plug is assumed to be located at the axial position $B_{p} / B_{b}=2$. The magnetic field axial profile is adopted to that of the GAMMA10 end-mirror cell. ${ }^{2}$ The magnitude of the potential $z=z_{m}$ must be given in advance in order to determine the potential profile. That is, the separatrix $\varepsilon=\mu B_{m}+e \phi_{m}$ is given at first to determine the loss cone in Fig. 1(b). So the different potential profiles in Fig. 2 correspond to the different boundary conditions of $\phi_{m}$, where $\phi_{m}$ is the $\phi$ at $z$ $=z_{m}$. The continuous potential is found to exist only in the range of $\phi_{m}$ given in Fig. 2 in the calculation. For example, when the magnitude of $e \phi_{m} / T_{i}$ is larger than 1.9 or is smaller than 1.3, the continuous potential is not obtained in Fig. 2(a).

The distribution function $f_{\text {ioni }}$ in (2) resulting from ionization is taken into account in Fig. 2. If there is no ionization around plug the potential jump (sheath potential) appears at $z=z_{p} .{ }^{5,6}$ Here we have assumed $T_{n i} / T_{i}=0.01$ in the calculation. The density of the ionized ions is extremely smaller than that of the passing and trapped ions so that the density profile does not change due to the inclusion of the effects of ionization. The height of the potential depends on the coefficients of $\alpha_{i}$ and $\alpha_{e}$ as well as the magnitude of $\phi_{m}$.

The required ionization rate for the formation of the continuous potential profile is shown in Fig. 3. As is seen in the figure the ionization rate is proportional to the quantity 


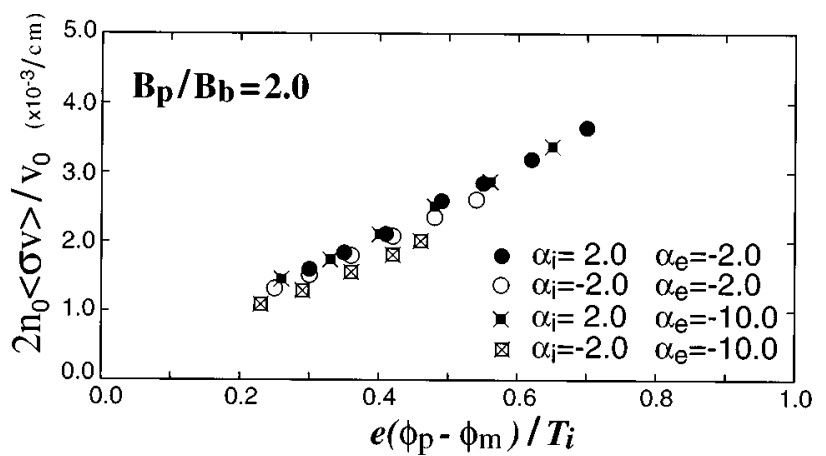

FIG. 3. The required minimum ionization rate for the continuous potential profile. Here $v_{0} \equiv \sqrt{2 T_{n i} / m_{i}}$. The different symbols in the figure correspond to the different parameters of distribution functions with $\alpha_{i}$ and with $\alpha_{e}$.

$e\left(\phi_{p}-\phi_{m}\right) / T_{i}$, but is almost independent of the coefficients $\alpha_{i}, \alpha_{e}$. The magnitudes of $B_{p}$ and $\phi_{p}$ are, of course, a function of $\alpha_{i}$ and $\alpha_{e}$, so that the required ionization rate should be a function of $\alpha_{i}$ and $\alpha_{e}$. The results in Fig. 3 indicate that the required ionization rate is a function of $e\left(\phi_{p}\left(\alpha_{i}, \alpha_{e}\right)-\phi_{m}\right) / T_{i}$ and $B_{p}\left(\alpha_{i}, \alpha_{e}\right) / B_{b}$.

The required ionization rate for the formation of the continuous potential profile for various $B_{p} / B_{b}$ is shown in Fig. 4. When the plug locates at a higher magnetic field, i.e., larger $B_{p} / B_{b}$, the larger ionization is required to avoid the sheath potential formation. This is due to the fact that the potential profile around the plug is flatter for the lower $B_{p} / B_{b}$.

Figure 5 shows the required ionization rate in the case of $B_{p} / B_{b}=2$. The different temperatures of the neutral atoms $T_{n i} / T_{i}$ are plotted in the figure. The lower temperature $T_{n i}$ requires the lower ionization rate for the continuous potential profile.

Now we estimate the required ionization rate in the GAMMA10 tandem mirror. ${ }^{2}$ As seen in Fig. 5 the condition $2 n_{0}\langle\sigma v\rangle / v_{0} \gtrsim 10^{-3} / \mathrm{cm}$ should be satisfied for the continuous potential formation around the plug in the case of

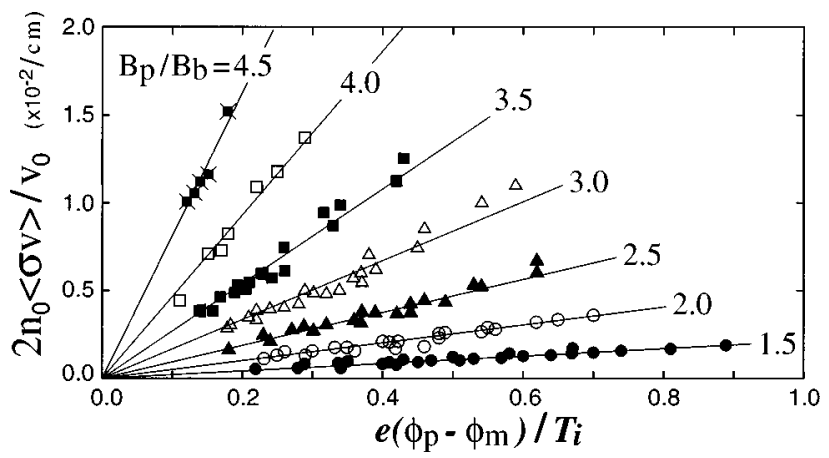

FIG. 4. The required minimum ionization rate for the continuous potential profiles. Here parameters of $\alpha_{i}=2.0,-2.0$ and $\alpha_{e}=-2.0,-10.0$ are plotted with the same symbol. The different symbols correspond to the different parameters of $B_{p} / B_{b}$. That is, solid circles are $B_{p} / B_{b}=1.5$, open circles are $B_{p} / B_{b}=2.0$, solid triangles are $B_{p} / B_{b}=2.5$, open triangles are $B_{p} / B_{b}$ $=3.0$, solid squares are $B_{p} / B_{b}=3.5$, open squares are $B_{p} / B_{b}=4.0$, and small solid squares with cross bars are $B_{p} / B_{b}=4.5$.

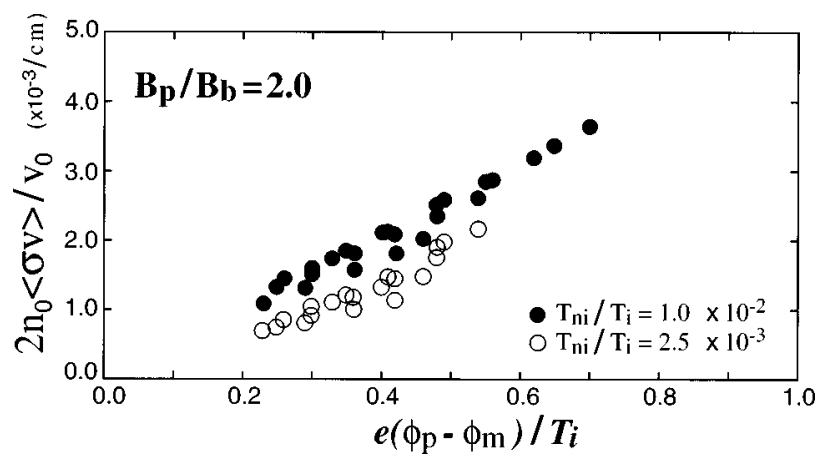

FIG. 5. The required minimum ionization rate for the continuous potential profiles. Here parameters of $\alpha_{i}=2.0,-2.0$ and $\alpha_{e}=-2.0,-10.0$ are plotted with the same symbol. Solid circles are $T_{n i} / T_{i}=1.0 \times 10^{-2}$ and open circles are $T_{n i} / T_{i}=2.5 \times 10^{-3}$.

$B_{p} / B_{b}=2$. That is, $n_{0}\langle\sigma v\rangle$ should be larger than $1.2 \times 10^{3} / \mathrm{s}$ with the assumption of $T_{n i}=3 \mathrm{eV}$ of hydrogen ions. Defining the ionization time $\tau_{\text {ioni }} \equiv 1 /\left(n_{0}\langle\sigma v\rangle\right)$, we obtain the condition $\tau_{\text {ioni }} \leq 8.3 \times 10^{-4} \mathrm{~s}$ for the continuous potential formation.

In summary it is found that the continuous plug potential profile can be formed by including the ionization source. The Emmert's source was adopted as an ionization term. The ionization time $\tau_{\text {ioni }} \leq 1.0 \times 10^{-3} \mathrm{~s}$ is necessary for the potential to be continuous across the plug, i.e., to be formed without the sheath potential formation. The required value of $\tau_{\text {ioni }}$, however, is one order magnitude shorter than that expected in GAMMA10.,10 A different mechanism playing the role of ionized ions, therefore, should be suggested. The ion distribution function in the dark painted region in Fig. 1, which is shown by an arrow with the comment of "continuous distribution function," is expected to be supplied, for example, by the oscillation of the plug potential.

${ }^{a}$ Electronic mail: katanuma@prc.tsukuba.ac.jp

${ }^{1}$ D. E. Baldwin and B. G. Logan, Phys. Rev. Lett. 43, 1318 (1979).

${ }^{2}$ T. Tamano, Phys. Plasmas 2, 2321 (1995).

${ }^{3}$ R. S. Post, K. Brau, J. Casey, X. Chen, J. Coleman, H. R. Garner, M. Gerver, S. Golovato, D. Goodman, W. Guss, S. Hiroe, S. Hokin, S. Horne, J. Irby, J. Kesner, B. Lane, T. Morgan, L. Pócs, E. Sevillano, D. Smatlak, D. K. Smith, J. Sullivan, R. P. Torti, and X. Z. Yao, in Proceedings of the 11th International Conference, Kyoto, 1986 (International Atomic Energy Agency, Vienna, 1987), Vol. 2, p. 251.

${ }^{4}$ I. Katanuma, Y. Kiwamoto, Y. Tatematsu, K. Ishii, T. Saito, K. Yatsu, and T. Tamano, Phys. Plasmas 3, 2218 (1996).

${ }^{5}$ T. Saito, I. Katanuma, T. Aota, L. G. Bruskin, T. Cho, M. Hirata, H. Hojo, M. Ichimura, K. Ishii, A. Itakura, N. Katsuragawa, Y. Kiwamoto, J. Kohagura, A. Mase, Y. Nakashima, Y. Sakamoto, T. Tamano, Y. Tatematsu, T. Tokuzawa, K. Yatsu, M. Yoshikawa, and Y. Yoshimura, in Proceedings of the 16th International Conference on Fusion Energy, Montréal, 1996 (International Atomic Energy Agency, Vienna, 1997), Vol. 2, p. 105.

${ }^{6}$ I. Katanuma, Y. Kiwamoto, Y. Tatematsu, K. Ishii, T. Saito, K. Yatsu, and T. Tamano, Phys. Plasmas 4, 2532 (1997).

${ }^{7}$ K.-U. Riemann, J. Phys. D 24, 493 (1991).

${ }^{8}$ J. Kesner, G. Knorr, and D. R. Nicholson, Nucl. Fusion 10, 1265 (1981).

${ }^{9}$ G. A. Emmert, R. M. Wieland, A. T. Mense, and J. N. Davidson, Phys. Fluids 23, 803 (1980).

${ }^{10}$ Y. Nakashima, M. Shoji, K. Yatsu, S. Kobayashi, K. Tsuchiya, N. Yamaguchi, M. Yoshikawa, T. Aota, T. Ishijima, M. Ichimura, M. Inutake, A. Mase, and T. Tamano, J. Nucl. Mater. 241-243, 1011 (1997). 